doi: $10.13108 / 2013-5-4-58$

\title{
MODIFIED GRADIENT FASTEST DESCENT METHOD FOR SOLVING LINEARIZED NON-STATIONARY NAVIER-STOKES EQUATIONS
}

\section{I.I. GOLICHEV}

\begin{abstract}
We introduce a regularization of Navier-Stokes equations, whose solution coincides with the solution to the system of Navier-Stokes equations if the latter exists. The regularized nonlinear system is reduced to solving a sequence of linearized systems. To solve the latter system, we employ the gradient method. We construct and justify a modified method of fastest descent, which may be employed under restrictions on the control and an unbounded Lebesgue set.
\end{abstract}

Keywords: Navier-Stokes equations, gradient method, regularization, apriori estimates.

Mathematics Subject Classification: 49M20, 35Q30, 93C05

\section{INTRODUCTION}

Consider the initial boundary value problem for the generalized system of Navier Stokes equations

$$
\begin{gathered}
\mathbf{v}_{t}-\nu \Delta \mathbf{v}+v_{i} \mathbf{v}_{x_{i}}+\operatorname{grad} p=\mathbf{f}(x, t), \\
\left.\mathbf{v}\right|_{S_{T}}=0,\left.\quad \mathbf{v}\right|_{t=0}=\mathbf{a}(x), \\
\operatorname{div} \mathbf{v}=0
\end{gathered}
$$

in the domain $Q_{T}=\Omega \times[0, T], S_{T}=S \times[0, T], S$ is the boundary of domain $\Omega, \mathbf{f} \in \stackrel{\circ}{\mathbf{J}}\left(Q_{T}\right)$, $\mathbf{L}_{2}\left(Q_{T}\right)=\mathbf{G}\left(Q_{T}\right) \oplus \stackrel{\mathbf{J}}{ }\left(Q_{T}\right)$ is the orthogonal decomposition on gradient and solenoidal parts of the space $\mathbf{L}_{2}\left(Q_{T}\right), \mathbf{v}=\left(v_{1}, v_{2}, \ldots, v_{n}\right)$,

$$
\operatorname{div} \mathbf{a}=0,\left.\quad \mathbf{a}\right|_{S}=0 .
$$

Hereinafter we employ the notations of work [3]. For the well-definiteness of the pressure we assume that $\int_{\Omega} p(x, t) d x=0$ for a.e. $t$ in $[0, T]$.

As it was mentioned in work [1], the main difficulty in studying the problem (1)-(3) is related with the global unique solvability, i.e., for each $t \in[0, T]$, of initial boundary value problem (1), (2). The justification of global solvability is hampered by the proof of an apriori estimate for one of the norms $\left\|\mathbf{v}_{x}(x, t)\right\|_{2},\|\mathbf{v}\|_{q, r, Q_{T}}$, where the parameters $q$ and $r$ satisfy certain conditions. The estimate on $\|\mathbf{v}\|_{q, r, Q_{T}}$ implies that for $\left\|\mathbf{v}_{x}(x, t)\right\|_{2}$ and vice-versa. Due to this situation, various regularizations of Navier-Stokes equations are considered, see, for instance, [1], [2] and the references therein. As a rule, the regularization is related with introducing additional terms with a small parameter into equation (1). At that, the solution to the regularized problem should converge to the solution of the initial Navier-Stokes problem as $\varepsilon \rightarrow 0$ provided it exists. Within such approach, there appear an issue on physical relevance of the regularized problem,

I.I. Golichev, Modified GRAdient FASTESt DESCEnt METHOD FOR SOlving LineARIZED NONSTATIONARY NAVIER-STOKES EQUATIONS.

(C) Golichev I.I. 2013.

Submitted December 3, 2013. 
on the choice of parameter $\varepsilon$ and on how the solutions of the regularized and original problem are close.

The approach for solving problem (1)-(3) suggested in work [3] can be also considered as the regularization of Navier-Stokes system. Its matter is that in the product $v_{i} \mathbf{v}_{x_{i}}$ in equation (1) the term $\mathbf{v}$ is replaced by its projection on the ball $K_{R}(t)=\left\{\mathbf{v}(t):\left\|\mathbf{v}_{x}(t)\right\| \leqslant R(t)\right\}$ for almost each $t$ in $[0, T]$, where $R(t)$ is a non-decreasing positive function. Hereinafter $\|\cdot\|=\|\cdot\|_{\mathbf{L}_{2}(\Omega)}$.

The projection on the ball $K_{R}$ is calculated by the formula $P_{K_{R}} \mathbf{v}=\alpha_{R}(t, \mathbf{v}) \mathbf{v}$, where $\alpha_{R}(t, \mathbf{v}(t))=\min \left[1, R(t) /\left\|\mathbf{v}_{x}(t)\right\|\right]$. Thus, from equation (1) we pass to the equation

$$
\mathbf{v}_{t}-\nu \Delta \mathbf{v}+\alpha_{R}(t, \mathbf{v}) v_{i} \mathbf{v}_{x_{i}}+\operatorname{grad} p=\mathbf{f} .
$$

For the solution of regularized problem (1) , (2), (3) we construct the iteration process

$$
\begin{gathered}
\mathbf{v}_{t}^{k+1}-\nu \Delta \mathbf{v}^{k+1}+\alpha_{R}^{k+1} v_{i}^{k} \mathbf{v}_{x_{i}}^{k+1}+\operatorname{grad} p^{k+1}=\mathbf{f}, \\
\left.\mathbf{v}^{k+1}\right|_{S_{T}}=0,\left.\quad \mathbf{v}^{k+1}\right|_{t=0}=\mathbf{a}(x), \\
\operatorname{div} \mathbf{v}^{k+1}=0,
\end{gathered}
$$

where $\alpha_{k}=\alpha_{k}(t)=\alpha_{R}\left(t, \mathbf{v}^{k}\right)$.

By $\mathbf{V}_{2}$ we denote the space $\mathbf{W}^{2,1}\left(Q_{T}\right) \cap \mathbf{L}_{\infty}\left(0, T ; \mathbf{W}_{2}^{1}(\Omega)\right)$ with the norm

$$
\|\mathbf{v}\|_{\mathbf{V}_{2}}=\|\mathbf{v}\|_{\mathbf{W}^{2,1}(Q)}+\underset{t \in[0, T]}{\operatorname{vraimax}}\left\|\mathbf{v}_{x}\right\|
$$

The following theorem was proven in [3]

Theorem 1. Let $\boldsymbol{f} \in \stackrel{\mathcal{J}}{ }\left(Q_{T}\right), \Omega$ be a bounded domain with the boundary $S \in C^{2}$, a(x) satisfy conditions (4). Then problem (1), (2), (3) has the unique solution $\boldsymbol{v}, p$ with $\boldsymbol{v}_{x x}, \boldsymbol{v}_{t}, p_{x}$ in $\boldsymbol{L}_{2}\left(Q_{T}\right)$ and sequences $\left\{\boldsymbol{v}^{k}\right\}_{k=0}^{\infty},\left\{p^{k}\right\}_{k=1}^{\infty}$ determined by the iteration process (5)-(7) converge to the solution of (1), (2), (3) for each $\boldsymbol{v}^{0} \in \boldsymbol{V}_{2}$. Here $\alpha_{k}=\min \left[1, R(t)\left\|\boldsymbol{v}_{x}^{k}\right\|^{-1}\right], R(t)$ is a bounded non-decreasing function. The estimates

$$
\begin{gathered}
\left\|\boldsymbol{v}^{k}-\boldsymbol{v}\right\|_{\boldsymbol{V}_{2}} \leqslant c(q) q^{k}\left\|\boldsymbol{v}^{0}-\boldsymbol{v}\right\|_{\boldsymbol{V}_{2}}, \\
\left\|p_{k}-p\right\|_{\boldsymbol{W}_{2}^{1,0}\left(Q_{T}\right)} \leqslant c(q) q^{k}\left\|\boldsymbol{v}^{0}-\boldsymbol{v}\right\|_{\boldsymbol{V}_{2}}
\end{gathered}
$$

hold true for each $q \in(0,1)$, where $c(q)$ is bounded on the segment $[\alpha, 1]$ for each $\alpha>0$.

It was also shown in [3] that the statement of Theorem 1 remains true if one replaces $\mathbf{v}(t)$ by its projection on the ball $\left\{\mathbf{v}(t) \in \mathbf{L}_{4}(\Omega):\|\mathbf{v}(t)\|_{4} \leqslant R(t)\right\}$ in the nonlinear term of equation (1); one can also replace by the coordinate projections of vector $\mathbf{v}(t)$ on the segment $\left[R_{1}(t), R_{2}(t)\right]$, where $R_{1}(t), R_{2}(t)$ are bounded on the interval $[0, T]$ functions.

Remark 1. We observe that the proven theorem ensures the convergence of iteration process (5)-(7) on each segment $[0, T]$ such that $\mathbf{f} \in \stackrel{\circ}{\mathbf{J}}\left(Q_{T}\right)$, as well as the unique solvability for problem (1), (2), (3).

Remark 2. If solution $\mathbf{v}^{*}, p_{*}$ of problem (1), (2), (3) satisfies the inequality

$$
\left\|\mathbf{v}_{x}^{*}(t)\right\| \leqslant R(t) \quad \forall t \in\left[0, T_{1}\right]
$$

on each interval $\left[0, T_{1}\right], T_{1} \leqslant T$, then the solution to problem (1)-(3) exists on this interval and $\mathbf{v}=\mathbf{v}^{*}, p=p_{*}$. Indeed, if inequality (11) holds true, then $\alpha\left(t, \mathbf{v}_{x}^{*}\right)=1$, and thus equations (1) and (í) coincide.

Remark 3. If the solution to problem (1)-(3) exists on the interval $\left[0, T_{1}\right], T_{1} \leqslant T$ and the estimate

$$
\left\|\mathbf{v}_{x}(t)\right\| \leqslant M(t) \quad \forall t \in\left[0, T_{1}\right]
$$

holds true, where $M(t) \leqslant R(t)$, then on this interval it coincides with the solution to regularized problem (1), (2), (3). Indeed, since $\mathbf{v}(\mathrm{t})$ lies inside the ball $K_{R}(t), \mathbf{v}(\mathrm{t})$ coincides with its projection on this ball and thus $\mathbf{v}$ satisfies equation (1). Taking into account the uniqueness 
of solution to problem (1), (2), (3) (by Theorem 1) and the uniqueness of solution to problem (1)-(3) under condition (12) (see [1, Ch. VI, Thm. 12 [1]), we arrive at the desired statement.

Remark 4. In view of Remark 2 it is easy to construct an iteration process converging to the solution of problem (1)-(3) in the case when the right hand side of estimate (12) is unknown under the condition that solution to problem (1)-(3) exists and satisfies restriction (12) for some unknown but bounded on $\left[0, T_{1}\right]$ function $M(t)$. Indeed, we take a positive bounded non-decreasing function $R_{1}(t)$ and solve equation (1), (2), (3) for $R(t)=R_{1}(t)$. Then we check condition (12) for $M(t)=R_{1}(t)$. If this condition holds, problem (1)-(3) is solved. If no, we let $R_{2}(t)=R_{1}(t)+K, K$ is the parameter of the method and repeat the iteration process. It is clear that after a final number of steps condition (12) will be satisfied and problem (1)-(3) will be thus solved.

While realizing the suggested approach, there appears an issue of the choice of integral restriction for $R(t)$ or uniform restrictions for the speed $R_{1}(t), R_{2}(t)$. The estimates for $\left\|\mathbf{v}_{x}(t)\right\|$ and $\|\mathbf{v}(t)\|_{4}$ can be explicitly found globally for $n=2$ and locally for $n=3$. These estimates on the interval $\left[t_{0}, t\right]$ depends on the initial condition $\left\|\mathbf{u}_{x}\left(t_{0}\right)\right\|\left(\left\|\mathbf{u}\left(t_{0}\right)\right\|_{4}\right),\|f\|_{\mathbf{L}_{2}\left(Q_{t_{0}}^{t}\right)}, \nu$ and the constants from embedding theorems.

In many cases it is difficult to find such estimates, moreover, applying them to a particular problem we can obtain very rough estimates. Because of this fact, in Remark 4 we suggest the iteration process which allows to find an apriori estimate if the solution with an appropriate estimate on a given time interval exists.

An apriori estimate of the form $|\mathbf{v}| \leqslant N$ can be prescribed by physical reasons if we know apriori that the speed of a viscous liquid does not exceeds a given quantity, i.e., $|\mathbf{v}(t, x)| \leqslant N$, then we can let $R_{1}(t)=-N, R_{2}(t)=N$. Then we note that if the solution of such regularized problem satisfies the chosen apriori estimate, then its solution coincides with the solution of the original problem. If the obtained solution does not satisfy the chosen estimate, then either the speed is estimated in a wrong way, of the original model (1) - (3) is irrelevant for the studied physical process.

It follows from the said above that in many cases solving nonlinear Navier-Stokes system can be reduced to solving a sequence of linear problems.

There are various approaches for solving linear problems. Among them we mention one based on gradient methods for minimization the functional $J(\mathbf{v})=\int_{Q_{T}}|\operatorname{div} \mathbf{v}|^{2} d x d t$, where pressure $p$ is treated as a control (see, for instance, [5]-[7]). However, while constructing the gradient method, one faces the difficulty related with the fact that in the considered problems (as in the most part of real problems, where the state of a system is described by differential equations) the Lebesgue sets $\mathbf{M}_{i}(C)=\left\{u \in U_{i}: J_{i}(u)<C, i=1,2\right\}$ are unbounded. In work [5] this difficulty was overcome by means of iterative regularization of the gradient projection method. Unfortunately, this method converges too slowly.

In the present work we construct and justify a modified method of fastest descent which can be applied under some restrictions for the control and unboundedness of the Lebesgue set.

\section{GRADIENT METHOD FOR SOLVING LINEARIZED PROBLEM}

It was shown in the previous section that under certain conditions solving of problem (1.1)(1.3) is reduced to solving the sequence of problems (1.5)-(1.7). Omitting index $k$, we write this problem as

$$
\begin{gathered}
L \mathbf{v} \equiv \mathbf{v}_{t}-\nu \Delta \mathbf{v}+g_{i} \mathbf{v}_{x_{i}}=\mathbf{f}-\operatorname{grad} p \\
\left.\mathbf{v}\right|_{S_{T}}=0,\left.\quad \mathbf{v}\right|_{t=0}=\mathbf{a}(x) \\
\operatorname{div} \mathbf{v}=0
\end{gathered}
$$


where $\mathbf{f} \in \stackrel{\circ}{\mathbf{J}}\left(Q_{T}\right), g_{i} \in L_{4, \infty}\left(Q_{T}\right)$, a $(x)$ satisfies condition (1.4). Hereinafter while referring to the formulae from another section, we make use of a double numeration, where the first number indicates the number of the section, and the other stands for the number of the formula within the section.

We consider problem (1)-(3) as the inverse problem of determining $\mathbf{v}$ and $p$ by additional data (3). The main aim of solving inverse problems is their reduction to problems of optimal control. We consider two versions of such problems.

Problem I. Find the minimum of the functional $J_{1}(u)=\frac{1}{2} \int_{Q_{T}}|\operatorname{div} \mathbf{v}(\nabla u)|^{2} d x d t$ on the set $U_{1}=\stackrel{\circ}{W}_{2}^{1,0}\left(Q_{T}\right)=\left\{u \in W_{2}^{1,0}\left(Q_{T}\right): \int_{\Omega} u(x, t) d x=0, t \in[0, T]\right\}$, where $\nabla u=\operatorname{grad} u, \mathbf{v}(\nabla u)$ solves problem $L \mathbf{v}=\mathbf{f}-\nabla u$ with conditions (2).

Problem II. Find the minimum of the functional $J_{2}(u)=\frac{1}{2} \int_{Q_{T}}|\operatorname{div} \mathbf{v}(\nabla u)|^{2} d x d t$ on the set $U_{2}=\stackrel{\circ}{L_{2}}\left(Q_{T}\right)=\left\{u \in L_{2}\left(Q_{T}\right): \int_{\Omega} u(x, t) d x=0, ; t \in[0, T]\right\}$, where $\mathbf{v}(\nabla u)$ solves problem $L \mathbf{v}=\mathbf{f}-\nabla u$ with condition (2).

The difference between problems II and I is that the derivatives $\nabla u$ for $u \in L_{2}\left(Q_{T}\right)$ are treated in the generalized sense and solution to problem (1) - (3) will be also treated in the generalized sense.

The we denote by $H_{l}, l=1,2$ the Hilbert spaces

$$
H_{1}=W_{2}^{1,0}\left(Q_{T}\right), H_{2}=\mathbf{L}_{2}\left(Q_{T}\right) ;
$$

then $U_{l}$ is a subspace of space $H_{l}, l=1,2$.

We solve problems I, II by the gradient projection method

$$
u_{k+1}=P_{U_{l}}\left(u_{k}-\alpha_{k+1} J_{l}^{\prime}\left(u_{k}\right)\right),
$$

where $P_{U_{l}}$ the projector on set $U_{l}, J_{l}^{\prime}\left(u_{k}\right)$ is the gradient of the functional $J_{l}\left(u_{k}\right)$ at the point $u_{k}, l=1,2$.

It will be shown in the next subsection that the formulae for calculating gradients

$$
J_{1}^{\prime}(u)=-p(u)
$$

hold true, where $p(u)$ is determined by the decomposition $\mathbf{w}(u)$ on gradient and solenoidal parts $\mathbf{w}(u)=\operatorname{grad} p(u)+\varphi$,

$$
J_{2}^{\prime}(u)=\operatorname{div} \mathbf{w}(u) .
$$

Here $\mathbf{w}(u)$ is the adjoint condition determined for both the problems as the solution to the problem

$$
\begin{gathered}
L^{*} \mathbf{w}(u)=-\mathbf{w}_{t}-\nu \Delta \mathbf{w}-\frac{\partial}{\partial x_{i}}\left(g_{i} \mathbf{w}\right)=\operatorname{grad} \operatorname{div} \mathbf{v}(\nabla u), \\
\left.\mathbf{w}\right|_{S_{T}}=0, \mathbf{w}(x, T)=0 .
\end{gathered}
$$

2.1. Differentiability of functional $J_{1}(u)$. We consider first the problem I. It is written as

$$
J_{1}(u)=\frac{1}{2} \int_{Q_{T}}|\operatorname{div} \mathbf{v}(\nabla u)|^{2} d x d t \rightarrow i n f ; u \in U_{1},
$$

where $\mathbf{v}(\nabla u)$ is the solution to equation

$$
L \mathbf{v}=\mathbf{v}_{t}-\nu \Delta \mathbf{v}+g_{i} \mathbf{v}_{x_{i}}=\mathbf{f}-\nabla u
$$

with initial and boundary conditions (2).

The proof of existence of solution to problem (1), (2) in space $\mathbf{W}_{2}^{2,1}\left(Q_{T}\right)$ and estimates necessary for justifying formula $\left(5_{1}\right)$ are based on the following lemma. 
Lemma 1. Let $\boldsymbol{F}(x, t) \in \boldsymbol{L}_{2}\left(Q_{T}\right), \boldsymbol{g}=\left(g_{1}, \ldots, g_{n}\right) \in \boldsymbol{L}_{4, \infty}\left(Q_{T}\right), \quad r \in L_{4, \infty}\left(Q_{T}\right)$, $\boldsymbol{a}(x) \in \boldsymbol{W}_{2}^{1}(\Omega)$. Then in the space $\boldsymbol{W}_{2}^{2,1}(\Omega)$ there exists the unique solution to the equation

$$
L(\boldsymbol{v})+r \boldsymbol{v}=\boldsymbol{F}, \quad(x, t) \in Q_{T},
$$

with initial and boundary conditions (2). The estimate

$$
\begin{aligned}
\|\boldsymbol{v}\|_{\lambda}^{2} & \equiv \underset{t \in[0, T]}{\operatorname{vraiamax}}\left\|\boldsymbol{v}_{x}(t)\right\|^{2}+\nu \int_{0}^{T}\|\Delta \boldsymbol{v}\|^{2} d t+\lambda \int_{0}^{T}\left\|\boldsymbol{v}_{x}(t)\right\|^{2} d t \\
& \leqslant 4 e^{\lambda T}\left(\nu^{-1}\|\boldsymbol{F}\|_{L_{2}\left(Q_{T}\right)}^{2}+\frac{1}{2}\|\boldsymbol{a}(x)\|^{2}\right)
\end{aligned}
$$

is valid, where $\lambda$ is a constant depending only on $\nu$ and constants $c_{2}, c_{3}, c_{4}, c_{7}$ from the embedding theorems and the second energy inequality (see inequalities (13)-(15), (20) in work [3]), $\|\boldsymbol{g}\|_{L_{4, \infty}\left(Q_{T}\right)},\|r\|_{\boldsymbol{L}_{4, \infty}\left(Q_{T}\right)}$.

Proof. We choose a sequence of bounded on $Q_{T}$ functions $\left\{\mathbf{F}^{n}\right\},\left\{\mathbf{g}^{n}\right\},\left\{r^{n}\right\}$ satisfying the conditions

$$
\begin{aligned}
\lim _{n \rightarrow \infty}\left\|\mathbf{F}^{n}-\mathbf{F}\right\|_{\mathbf{L}_{2}\left(Q_{T}\right)} & =\lim _{n \rightarrow \infty}\left\|\mathbf{g}^{n}-\mathbf{g}\right\|_{\mathbf{L}_{4, \infty}\left(Q_{T}\right)} \\
& =\lim _{n \rightarrow \infty}\left\|r^{n}-r\right\|_{L_{4, \infty}\left(Q_{T}\right)}=0
\end{aligned}
$$

and consider the sequence of the problems

$$
\begin{gathered}
\mathbf{v}_{t}^{n}-\nu \Delta \mathbf{v}^{n}+g_{i}^{n} \mathbf{v}_{x_{i}}^{n}+r^{n} \mathbf{v}=\mathbf{F}^{n}, \\
\left.\mathbf{v}^{n}\right|_{S_{T}}=0,\left.\quad \mathbf{v}^{n}\right|_{t=0}=\mathbf{a} .
\end{gathered}
$$

We note that the latter problem splits into separate problems for the coordinates of vector $\mathbf{v}^{n}$. Employing the known results (see, for instance, [4, Ch. III, Sec. 6]), we make sure that $\left\|\mathbf{v}^{n}\right\|_{\lambda}$ is bounded. Let us prove the uniform estimate $\left\|\mathbf{v}^{n}\right\|_{\lambda} \leqslant c_{0}, n=1,2, \ldots$ $\lambda$.

In what follows by $C_{i}$ we denote the constants depending on the same quantities as constant We denote $\widetilde{\mathbf{v}}^{n}=\mathbf{v}^{n} e^{-\lambda t} ; \widetilde{\mathbf{F}}^{n}=\mathbf{F}^{n} e^{-\lambda t}$, then $\widetilde{\mathbf{v}}^{n}$ is a solution to the problem

$$
\begin{gathered}
\widetilde{\mathbf{v}}_{t}^{n}-\nu \Delta \widetilde{\mathbf{v}}^{n}+g_{i}^{n} \widetilde{\mathbf{v}}_{x_{i}}^{n}+r^{n} \widetilde{\mathbf{v}}^{n}+\lambda \widetilde{\mathbf{v}}^{n}=\widetilde{F}^{n}, \\
\left.\widetilde{\mathbf{v}}^{n}\right|_{S_{T}}=0,\left.\quad \widetilde{\mathbf{v}}^{n}\right|_{t=0}=\mathbf{a} .
\end{gathered}
$$

We multiply equation $\left(13^{\prime}\right)$ by $\Delta \widetilde{\mathbf{v}}$ and integrating by parts over domain $Q_{t}$, we obtain

$$
\begin{gathered}
\frac{1}{2}\left\|\widetilde{\mathbf{v}}_{x}^{n}(t)\right\|^{2}+\nu\left\|\left|\Delta \widetilde{\mathbf{v}}^{n}\right|\right\|_{0, t}^{2}+\left(\left(g_{i}^{n} \widetilde{\mathbf{v}}_{x_{i}}+r^{n} \widetilde{\mathbf{v}}^{n}\right), \Delta \widetilde{\mathbf{v}}^{n}\right)_{\mathbf{L}_{2}\left(Q_{T}\right)}+\lambda\left\|\left|\widetilde{\mathbf{v}}_{x}^{n}(t)\right|\right\|_{0, t}^{2} \\
=\left(\widetilde{\mathbf{F}}^{n}, \Delta \widetilde{\mathbf{v}}^{n}\right)_{\mathbf{L}_{2}\left(Q_{T}\right)}+\frac{1}{2}\left\|\mathbf{a}_{x}\right\|^{2} .
\end{gathered}
$$

Here we have used the notation $\||\cdot|\|_{0, t}=\|\cdot\|_{L_{2}\left(Q_{t}\right)}$.

It follows from relations (12) that there exist constants $C_{1}, C_{2}, C_{3}$ such that the estimates

$$
\left\|\left|\mathbf{F}^{n}\right|\right\|_{0, t} \leqslant C_{1},\left\|\mathbf{g}^{n}(t)\right\|_{\mathbf{L}_{4}(\Omega)} \leqslant C_{2},\left\|r^{n}(t)\right\|_{L_{4}(\Omega)} \leqslant C_{3} \quad \forall t \in[0, T]
$$

are valid. 
To estimate the integrals in the left hand side of identity (15), we make use of the following relations

$$
\begin{aligned}
\left\|\left|g_{i}^{n} \widetilde{\mathbf{v}}_{x_{i}}^{n}\right|\right\|_{0, t} & \leqslant\left(\int_{0}^{t} \int_{\Omega}\left|\mathbf{g}^{n}\right|^{2}\left|\widetilde{\mathbf{v}}_{x}^{n}\right|^{2} d x d t\right)^{\frac{1}{2}} \leqslant\left(\int_{0}^{t}\left\|\mathbf{g}^{n}\right\|_{4}^{2}\left\|\widetilde{\mathbf{v}}_{x}^{n}\right\|_{4}^{2} d \tau\right)^{\frac{1}{2}} \\
& \leqslant C_{2}\left(\int_{0}^{t}\left\|\widetilde{\mathbf{v}}_{x}^{n}\right\|_{4}^{2} d \tau\right)^{\frac{1}{2}} \leqslant \varepsilon\left\|\Delta \widetilde{\mathbf{v}}^{n}\right\|_{0, t}+c(\varepsilon)\left\|\Delta \widetilde{\mathbf{v}}_{x}^{n}\right\|_{0, t} .
\end{aligned}
$$

Here we have used the inequality

$$
\left(\int_{0}^{t}\left\|\mathbf{w}_{x}\right\|_{4}^{2} d \tau\right)^{\frac{1}{2}} \leqslant \varepsilon\||\Delta \mathbf{w}|\|_{0, t}+c(\varepsilon)\left\|\left|\mathbf{w}_{x}\right|\right\|_{0, t}
$$

which is valid for each $w \in \stackrel{\circ}{W}_{2}^{2,1}\left(Q_{T}\right)$.

The latter inequality can be obtained, for instance, for $n=3$, as follows:

$$
\int_{0}^{t}\left\|\mathbf{w}_{x}\right\|_{2}^{4} d \tau \leqslant c_{3} c_{7}\left(\int_{0}^{t}\left\|\mathbf{w}_{x}\right\|^{\frac{1}{2}}\|\Delta \mathbf{w}\|^{\frac{3}{4}} d \tau\right)^{\frac{1}{2}} \leqslant\left. c_{3} c_{7}\left\|\left|\mathbf{w}_{x}\left\|\left.\right|_{0, t} ^{\frac{1}{4}}\right\|\right| \Delta \mathbf{w}\right\|\right|_{0, t} ^{\frac{3}{4}} .
$$

Here we have used the estimate from the embedding theorem $\|v\|_{4} \leqslant c_{3}\left\|v_{x}\right\|^{\frac{3}{4}}\|v\|^{\frac{1}{4}}$ (as $n=3$ ) and the second energy estimate $\left\|v_{x x}\right\| \leqslant c_{7}\|\Delta v\|$ being valid for $\stackrel{\mathbf{W}}{2}_{2}^{1}(\Omega) \cap \mathbf{W}_{2}^{2}(\Omega)$. Employing then Young's inequality $\left(a b \leqslant \frac{1}{m} \varepsilon_{1}^{m} a^{m}+\frac{m-1}{m} \varepsilon_{1}^{-\frac{m-1}{m}} b^{\frac{m}{m-1}}\right)$, where $m=\frac{4}{3}$, we obtain inequality (17).

Taking into consideration estimates (16) and the estimates $\|\mathbf{v}\|_{4} \leqslant \bar{c}\left\|\mathbf{v}_{x}\right\|$ for $n=2$, 3 , it is easy to make sure that the following inequalities

$$
\left\|\left|r^{n} \widetilde{\mathbf{v}}^{n}\right|\right\|_{0, t} \leqslant\left(\int_{0}^{t}\left\|r^{n}\right\|_{4}^{2}\left\|\widetilde{\mathbf{v}}^{n}\right\|_{4}^{2} d \tau\right)^{\frac{1}{2}} \leqslant C_{3} \bar{c}\left\|\left|\widetilde{\mathbf{v}}_{x}^{n}\right|\right\|_{0, t}
$$

are valid. They imply

$$
\begin{aligned}
A_{1} & =\left|\left(\left(g_{i}^{n} \widetilde{\mathbf{v}}_{x_{i}}^{n}+r^{n} \widetilde{\mathbf{v}}^{n}\right), \Delta \widetilde{\mathbf{v}}^{n}\right)_{\mathbf{L}_{2}\left(Q_{t}\right)}\right| \\
& \leqslant\left(\varepsilon\left\|\left|\Delta \widetilde{\mathbf{v}}^{n}\right|\right\|_{0, t}+\left(c(\varepsilon)+C_{3} \bar{c}\right)\left\|\left|\widetilde{\mathbf{v}}_{x}^{n}\right|\right\|_{0, t}\right)\left\|\left|\Delta \widetilde{\mathbf{v}}^{n}\right|\right\|_{0, t} \\
& \leqslant 2 \varepsilon\left\|\left|\Delta \widetilde{\mathbf{v}}^{n}\right|\right\|_{0, t}^{2}+\frac{1}{4 \varepsilon}\left(c(\varepsilon)+C_{3} \bar{c}\right)^{2}\left\|\left|\widetilde{\mathbf{v}}_{x}^{n}\right|\right\|_{0, t}^{2} .
\end{aligned}
$$

Letting $\varepsilon=\frac{1}{8} \nu$, and $\lambda=4 \nu^{-1}\left(c\left(\frac{\nu}{8}\right)+C_{3} \bar{c}\right)^{2}$, we obtain

$$
A_{1} \leqslant \frac{1}{4} \nu\left\|\left|\Delta \widetilde{\mathbf{v}}^{n}\right|\right\|_{0, t}^{2}+\frac{1}{2} \lambda\left\|\left|\widetilde{\mathbf{v}}_{x}^{n}\right|\right\|_{0, t} .
$$

Taking into consideration the latter inequality, relation (15) and inequality

$$
A_{2}=\left|\left(\widetilde{\mathbf{F}}^{n}, \Delta \widetilde{\mathbf{v}}^{n}\right)_{\mathbf{L}_{2}\left(Q_{T}\right)}\right| \leqslant \frac{1}{4} \nu\left\|\left|\widetilde{\Delta \mathbf{v}}^{n}\right|\right\|_{0, t}^{2}+\nu^{-1}\left\|\left|\widetilde{\mathbf{F}}^{n}\right|\right\|_{0, t},
$$

we obtain

$$
\frac{1}{2}\left\|\widetilde{\mathbf{v}}_{x}^{n}(t)\right\|^{2}+\frac{1}{2} \nu\left\|\left|\Delta \widetilde{\mathbf{v}}^{n}\right|\right\|_{0, t}^{2}+\frac{1}{2} \lambda\left\|\left|\widetilde{\mathbf{v}}_{x}^{n}\right|\right\|_{0, t}^{2} \leqslant c_{t}^{2},
$$


where $c_{t}^{2}=\nu^{-1}\left\|\left|\widetilde{\mathbf{F}}^{n}\right|\right\|_{0, t}^{2}+\frac{1}{2}\left\|\mathbf{a}_{x}\right\|^{2}$. By the latter inequality we find that

$$
\left[\widetilde{\mathbf{v}}^{n}\right]_{\lambda, t}^{2}=\underset{\tau \in[0, t]}{\operatorname{vraimax}}\left\|\widetilde{\mathbf{v}}^{n}(\tau)\right\|^{2}+\nu\left\|\left|\Delta \widetilde{\mathbf{v}}^{n}\right|\right\|_{0, t}^{2}+\lambda\|\| \widetilde{\mathbf{v}}_{x}^{n} \mid \|_{0, t}^{2} \leqslant 4 c_{t}^{2} .
$$

Let us show that the sequence $\left\{\widetilde{\mathbf{v}}^{n}\right\}$ is fundamental in the metrics $[\cdot]_{\lambda, T}=\|\cdot\|_{\lambda}$.

We denote $\mathbf{z}^{n, l}=\widetilde{\mathbf{v}}^{n}-\widetilde{\mathbf{v}}^{n+l}$ and note that $\mathbf{z}^{n, l}$ satisfies the equation

$$
\begin{aligned}
\mathbf{z}_{t}^{n, l} & -\nu \Delta \mathbf{z}^{n, l}+g_{i}^{n} \mathbf{z}_{x_{i}}^{n, l}+r^{n} \mathbf{z}^{n, l}+\lambda \mathbf{z}^{n, l} \\
& =\left(\widetilde{\mathbf{F}}^{n}-\widetilde{\mathbf{F}}^{n+l}\right)+\left(g_{i}^{n+l}-g_{i}^{n}\right) \widetilde{\mathbf{v}}_{x_{i}}^{n+l}+\left(r^{n+l}-r^{n}\right) \widetilde{\mathbf{v}}^{n+l}
\end{aligned}
$$

and the conditions

$$
\left.\mathbf{z}^{n, l}\right|_{S_{T}}=0,\left.\mathbf{z}^{n, l}\right|_{t=0}=0 .
$$

We can apply inequality (19) to problem (20), (21) with

$$
c_{t}^{2}=c_{t}^{2}(n, l)=\nu^{-1}\left(\left\|\left|\left(\widetilde{\mathbf{F}}^{n}-\widetilde{\mathbf{F}}^{n+l}\right)+\left(g_{i}^{n+l}-g_{i}^{n}\right) \widetilde{\mathbf{v}}_{x_{i}}^{n+l}+\left(r^{n+l}-r^{n}\right) \widetilde{\mathbf{v}}^{n+l}\right|\right\|_{0, t}^{2}\right) .
$$

Bearing in mind condition (12), the boundedness of the sequence $\left\{\widetilde{\mathbf{v}}^{n}\right\}$ in the metrics $[\cdot]_{\lambda, T}$ and inequality (17), we obtain the estimates

$$
\begin{aligned}
& \left\|\left|\left(\mathbf{g}^{n+l}-\mathbf{g}^{n}\right) \widetilde{\mathbf{v}}^{n+l}\right|\right\|_{0, T} \leqslant C_{5}\left\|\mathbf{g}^{n+l}-\mathbf{g}^{n}\right\|_{\mathbf{L}_{4, \infty}\left(Q_{T}\right)}, \\
& \left\|\left|\left(r^{n+l}-r^{n}\right) \widetilde{\mathbf{v}}^{n+l}\right|\right\|_{0, T} .
\end{aligned}
$$

These inequalities, conditions (12), and estimates (19) yield the convergence of sequence $\left\{\widetilde{\mathbf{v}}^{n}\right\}$ in metrics $[\cdot]_{\lambda, T}$.

It is easy to show that $\lim _{n \rightarrow \infty} g_{i}^{n} \widetilde{\mathbf{v}}_{x_{i}}^{n}=g_{i} \widetilde{\mathbf{v}}_{x_{i}}, \lim _{n \rightarrow \infty} r^{n} \widetilde{\mathbf{v}}^{n}=r \widetilde{\mathbf{v}}$. Then it follows from equation (13) that $\left\{\widetilde{\mathbf{v}}_{t}^{n}\right\}_{n=0}^{\infty}$ converges in $\mathbf{L}_{2}\left(Q_{T}\right)$ to $\widetilde{\mathbf{v}}_{t} . \widetilde{\mathbf{v}} \in \mathbf{W}_{2}^{2,1}\left(Q_{T}\right)$.

Passing to the limit as $n \rightarrow \infty$ in inequality and taking into consideration the obvious inequalities

$$
\begin{array}{ll}
\|\widetilde{\mathbf{v}}(t)\| \geqslant e^{-\lambda t}\|\mathbf{v}(t)\|, \quad\left\|\widetilde{\mathbf{v}}_{x}(t)\right\| \geqslant e^{-\lambda t}\left\|\mathbf{v}_{x}(t)\right\| \\
\|\Delta \widetilde{\mathbf{v}}(t)\| \geqslant e^{-\lambda t}\|\Delta \mathbf{v}(t)\|, \quad\|\widetilde{\mathbf{F}}\|_{\mathbf{L}_{2}\left(Q_{T}\right)} \leqslant\|\mathbf{F}\|_{\mathbf{L}_{2}\left(Q_{T}\right)},
\end{array}
$$

we arrive at inequality (11). In its turn, it implies the uniqueness of solution to equation (10).

Corollary 1. Let $\boldsymbol{f} \in \boldsymbol{L}_{2}\left(Q_{T}\right), \boldsymbol{g} \in \boldsymbol{L}_{4, \infty}\left(Q_{T}\right), \boldsymbol{a}(x) \in \stackrel{\circ}{\boldsymbol{W}}_{2}^{1}(\Omega), S \in C^{2}$. Then for each $u \in W_{2}^{1,0}\left(Q_{T}\right)$ the equation

$$
L \boldsymbol{v}=\boldsymbol{f}-\operatorname{grad} u
$$

with boundary and initial conditions (2) the unique solution in $\boldsymbol{W}_{2}^{2,1}\left(Q_{T}\right)$ and the estimate

$$
\|\boldsymbol{v}\|_{\lambda} \leqslant C_{7} e^{\lambda T}\left(\|\boldsymbol{f}\|_{\boldsymbol{L}_{2}\left(Q_{T}\right)}+\|\operatorname{grad} u\|_{\boldsymbol{L}_{2}\left(Q_{T}\right)}+\left\|\boldsymbol{a}_{x}\right\|\right)
$$

holds true.

Corollary 2. Suppose that the hypothesis of Corollary 1 holds and in addition div $\boldsymbol{g} \in$ $\boldsymbol{L}_{4, \infty}\left(Q_{T}\right)$. Then for each $u \in W_{2}^{1,0}\left(Q_{T}\right)$ problem (6), (7) has a solution in $\boldsymbol{W}_{2}^{2,1}(\Omega)$ and the estimate

$$
\|\boldsymbol{w}\|_{\lambda} \leqslant C_{8} e^{\lambda T}\|\operatorname{grad} \operatorname{div} \boldsymbol{v}(\nabla u)\|_{L_{2}\left(Q_{T}\right)}
$$

holds true. 
Proof. First we note that in Lemma $1, \mathbf{g}$ is an arbitrary function in $\mathbf{L}_{4, \infty}\left(Q_{T}\right)$. Writing the left hand side of equation (6) as

$$
L^{*} \mathbf{w} \equiv-\mathbf{w}_{t}-\nu \Delta \mathbf{w}-\mathbf{g}_{i} \mathbf{w}_{x_{i}}-\operatorname{div} \mathbf{g w}
$$

and making the change $t=T-\tau$, we pass to equation (10) with an arbitrary right hand side $F \in \mathbf{L}_{2}\left(Q_{T}\right)$ and homogeneous boundary and initial conditions. Employing inequality (11), we obtain estimate (23).

Corollary 3. Let a linear operator $L$ is defined by the differential expression $L \boldsymbol{v}=\boldsymbol{v}_{t}-$ $\nu \Delta \boldsymbol{v}+\boldsymbol{g}_{i} \boldsymbol{v}_{x_{i}}\left(g \in \boldsymbol{L}_{4, \infty}\left(Q_{T}\right)\right)$ on the set of functions $D(L) \subset \boldsymbol{W}_{2}^{2,1}\left(Q_{T}\right)$ satisfying homogeneous initial and boundary conditions (2). Then operator $L$ is closed, has a bounded inverse and its domain is $R(L)=\boldsymbol{L}_{2}\left(Q_{T}\right)$.

First two statements follow immediately from Lemma 1, while the closedness follows from the first two properties of operator $L$. Similar statements are valid for operator $L^{*}$ defined by the differential expression in the right hand side of equation (6) on the set of functions $\mathbf{w} \in D\left(L^{*}\right) \subset \mathbf{W}_{2}^{2,1}\left(Q_{T}\right)$ satisfying conditions (7). Integrating by parts, one can make sure that $L^{*}$ is contained in the adjoint operator $\tilde{L}^{*}$ of $L$. The coincidence of the domains of operators $L^{*}$ and $\tilde{L}^{*}$ can be shown easily. Indeed, let $z \in D\left(\tilde{L}^{*}\right)$, then letting $f=\tilde{L}^{*} z$, we obtain the relations $(L x, z)=(x, f) \forall x \in D(L)$. On the other hand, there exists an element $w \in D\left(\tilde{L}^{*}\right)$ such that $\tilde{L}^{*} w=f$. Hence, $(L x, z-w)=0$ for each $x \in D(L)$. Letting $x=L^{-1}(z-w)$, we obtain identity $z=w$.

Theorem 2. Suppose the hypothesis of Corollary 2 of Lemma 1. Then functional $J_{1}(u)$ is differentiable in $\stackrel{\circ}{\boldsymbol{W}}_{2}^{1,0}\left(Q_{T}\right)=U_{1}$ and its gradient satisfies Lipshitz condition.

Proof. To prove formula $\left(5_{1}\right)$ on the set $U_{1}=\stackrel{\circ}{W}_{2}^{1,0}\left(Q_{T}\right)$, we introduce the scalar product equivalent to that in $W_{2}^{1,0}\left(Q_{T}\right)$

$$
(v, z)_{\dot{W}_{2}^{1,0}}=\int_{Q_{T}} v_{x_{i}} z_{x_{i}} d x d t=(\nabla \mathbf{v}, \nabla \mathbf{z})_{\mathbf{L}_{2}\left(Q_{T}\right)} .
$$

Then

$$
\begin{aligned}
J_{1}(u+h) & -J_{1}(u)=\frac{1}{2}\|\operatorname{div} \mathbf{v}(\nabla u+\nabla h)\|_{L_{2}\left(Q_{T}\right)}^{2}-\frac{1}{2}\|\operatorname{div} \mathbf{v}(\nabla u)\|_{L_{2}\left(Q_{T}\right)}^{2} \\
& =(\operatorname{div} \mathbf{v}(\nabla u), \operatorname{div} \stackrel{\circ}{\mathbf{v}}(\nabla h))_{L_{2}\left(Q_{T}\right)}+\frac{1}{2}\|\operatorname{div} \mathbf{v}(\nabla h)\|_{L_{2}\left(Q_{T}\right)}^{2} .
\end{aligned}
$$

By Corollary 1, operator $L$ has the inverse $L^{-1}$, and in particular, $L^{-1} \mathbf{h}=\stackrel{\circ}{\mathbf{v}}(h)$. Taking into consideration Corollary 2 of Lemma 1, we make sure that operator $L^{*}$ has inverse and $\left(L^{*}\right)^{-1} \operatorname{grad} \operatorname{div} u=\mathbf{w}$, where $\mathbf{w}$ solves problem (6), (7). By Corollary 3 of Lemma $1, L^{*}$ is the adjoint operator for $L$.

Employing the above introduced operator $L$, we transform the first term in the right hand side of the latter identity:

$$
\begin{aligned}
(\operatorname{div} \mathbf{v}(\nabla u), \operatorname{div} \stackrel{\mathbf{v}}{ }(\nabla h))_{L_{2}\left(Q_{T}\right)}=-\left(\left(L^{*}\right)^{-1} \nabla \operatorname{div} \mathbf{v}(\nabla u), \nabla h\right)_{\mathbf{L}_{2}\left(Q_{T}\right)} \\
=-\left(P_{G\left(Q_{T}\right)} \mathbf{w}(u), \nabla u\right)_{\mathbf{L}_{2}\left(Q_{T}\right)}=-(\nabla p(u), \nabla h)_{\mathbf{L}_{2}\left(Q_{T}\right)}=(-p(u), h)_{\dot{W}_{2}^{1,0}} .
\end{aligned}
$$

Taking into consideration inequality (22), we obtain the estimates

$$
\begin{aligned}
\|\operatorname{div} \stackrel{\circ}{\mathbf{v}}(\nabla h)\|_{L_{2}\left(Q_{T}\right)}^{2} & \leqslant 2\left\|\dot{\mathbf{v}}_{x}(\nabla h)\right\|_{\mathbf{L}_{2}\left(Q_{T}\right)}^{2} \leqslant 2 \lambda^{-1}\|\stackrel{\circ}{\mathbf{v}}(\nabla h)\|_{\lambda}^{2} \\
& \leqslant 2 \lambda^{-1} C_{7} e^{\lambda T}\|\nabla h\|_{\mathbf{L}_{2}\left(Q_{T}\right)}^{2}=2 \lambda^{-1} C_{7} e^{\lambda T}\|h\|_{\mathbf{W}_{2}^{1,0}}^{2} .
\end{aligned}
$$

This relation and (24), (25) imply identity $\left(5_{1}\right)$. 
Let us show that $J_{1}^{\prime}(u)$ satisfies Lipshitz condition. Let $u^{1}$ and $u^{2}$ belong to $\stackrel{\circ}{W}_{2}^{1,0}\left(Q_{T}\right)$, while $\mathbf{w}^{1}$ and $\mathbf{w}^{2}$ are the corresponding solution to problem (6), (7). Then

$$
\begin{gathered}
\left\|J_{1}^{\prime}\left(u^{1}\right)-J_{1}^{\prime}\left(u^{2}\right)\right\|_{\dot{W}_{2}^{1,0}\left(Q_{T}\right)}=\left\|p\left(u^{1}\right)-p\left(u^{2}\right)\right\|_{\hat{W}_{2}^{1,0}\left(Q_{T}\right)}= \\
=\left\|\nabla p\left(u^{1}\right)-\nabla p\left(u^{2}\right)\right\|_{\mathbf{L}_{2}\left(Q_{T}\right)}=\left\|P_{G\left(Q_{T}\right)} \mathbf{w}^{1}-P_{G\left(Q_{T}\right)} \mathbf{w}^{2}\right\|_{\mathbf{L}_{2}\left(Q_{T}\right)} \leqslant \\
\leqslant\left\|\mathbf{w}^{1}-\mathbf{w}^{2}\right\|_{\mathbf{L}_{2}\left(Q_{T}\right)} .
\end{gathered}
$$

We note that $\mathbf{w}=\mathbf{w}^{1}-\mathbf{w}^{2}$ solves problem (6), (7), where $u=u^{1}-u^{2}$.

Employing inequalities (23), (22) and $\|\mathbf{v}\| \leqslant c_{4}\left\|\mathbf{v}_{x}\right\|,\left\|\mathbf{v}_{x x}\right\| \leqslant c_{7}\|\Delta \mathbf{v}\|$ being valid for each $\mathbf{v} \in \dot{\mathbf{W}}_{2}^{1}(\Omega)$, we get

$$
\begin{aligned}
\|\mathbf{w}\|_{\mathbf{L}_{2}\left(Q_{T}\right)} & \leqslant c_{4}\left\|\mathbf{w}_{x}\right\|_{\mathbf{L}_{2}\left(Q_{T}\right)} \leqslant c_{4} \lambda^{-1}\|\mathbf{w}\|_{\lambda} \leqslant c_{4} \lambda^{-1} C_{8} e^{\lambda T}\left\|\nabla \operatorname{div} \mathbf{v}\left(\nabla\left(u^{1}-u^{2}\right)\right)\right\|_{\mathbf{L}_{2}\left(Q_{T}\right)} \\
& \leqslant \sqrt{n} c_{4} \lambda^{-1} c_{7} C_{8} e^{\lambda T}\left\|\Delta \mathbf{v}\left(u^{1}-u^{2}\right)\right\|_{\mathbf{L}_{2}\left(Q_{T}\right)} \\
& \leqslant \sqrt{n} c_{4} c_{7} C_{8} \lambda^{-1} \nu^{-\frac{1}{2}} e^{\lambda T}\left\|\mathbf{v}\left(\nabla\left(u^{1}-u^{2}\right)\right)\right\|_{\lambda} \\
& \leqslant \sqrt{n} c_{4} c_{7} C_{8} \nu^{-\frac{1}{2}} \lambda^{-1} C_{7} e^{\lambda T}\left\|\nabla\left(u^{1}-u^{2}\right)\right\|_{\mathbf{L}_{2}\left(Q_{T}\right)} \\
& =L_{1}\left\|u^{1}-u^{2}\right\|_{\mathbf{W}_{2}^{1,0}} .
\end{aligned}
$$

This inequality and (26) imply that the gradient $J_{1}^{\prime}(u)$ satisfies Lipshitz condition with the constant $L_{1}=\sqrt{n} c_{4} c_{7} C_{8} \nu^{-\frac{1}{2}} \lambda^{-1} C_{7} e^{\lambda T}$.

2.2. Differentiability of functional $J_{2}(u)$. In studying problem II we shall need generalized solutions to problem (1)-(3) in the Banach space $\mathbf{V}_{2}^{1,0}\left(Q_{T}\right)$ obtained as a closure of smooth functions vanishing in the vicinity of $S_{T}$ by the norm

$$
\|\mathbf{v}\|_{Q_{T}}=\max _{0 \leqslant t \leqslant T}\|\mathbf{v}(x, t)\|_{L_{2}(\Omega)}+\left\|\mathbf{v}_{x}\right\|_{\mathbf{L}_{2}\left(Q_{T}\right)} .
$$

A generalized solution in the class $\dot{V}_{2}^{1,0}\left(Q_{T}\right)$ to the problem (1)-(3) is a function $\mathbf{v} \in \stackrel{\circ}{V}_{2}^{1,0} \cap \stackrel{\circ}{J}\left(Q_{T}\right)$ satisfying the identity

$$
\begin{aligned}
& \int_{Q_{T}}\left(-\mathbf{v} \Phi_{t}+\nu \mathbf{v}_{x} \Phi_{x}\right) d x d \tau+\int_{\Omega} \mathbf{v}(x, t) \Phi(x, t) d x+\int_{Q_{T}} q_{i} \mathbf{v}_{x_{i}} \Phi d x d \tau \\
& \quad=\int_{\Omega} \mathbf{a}(x) \Phi(x, 0) d x+\int_{Q_{T}} f \Phi d x d \tau, \quad t \in(0, T)
\end{aligned}
$$

for each $\Phi \in \dot{\mathbf{W}}_{2}^{1,1}\left(Q_{T}\right) \cap \stackrel{\circ}{J}\left(Q_{T}\right)$ and the identity

$$
\frac{1}{2}\|\mathbf{v}(x, t)\|^{2}+\nu \int_{0}^{t}\left\|\mathbf{v}_{x}\right\|^{2} d \tau=\int_{0}^{t}(f, \mathbf{v}) d \tau+\frac{1}{2}\|\mathbf{a}\|^{2}+\int_{Q_{T}} \operatorname{div} g\|\mathbf{v}\|^{2} d x d \tau .
$$

If the hypothesis of Theorem 2 holds, then solution to problem (1)-(3) obviously satisfies relations (27), (28), and this is why the solution to the generalized problem exists.

We note that if $S \in C^{2}$, it is easy to prove the unique solvability for problem (1)-(3) in $\stackrel{\circ}{\mathbf{V}}_{2}^{1,0}\left(Q_{T}\right)$ under the condition $\mathbf{a} \in \stackrel{\circ}{J}(\Omega), f \in \mathbf{L}_{2}\left(Q_{T}\right)$.

It can be done by passing to a limit in the sequence of the problems where a $\in \dot{J}(\Omega)$ is replaced by a sequence of smooth functions $\mathbf{a}_{n}$ in $H(\Omega)$ converging in the norm of $L_{2}(\Omega)$ (see, for instance, [1, Ch. IV, Thm. 3]).

To prove formula $\left(5_{2}\right)$ and to check Lipshitz condition for the gradient $J_{2}^{\prime}(u)$ of the functional $J_{2}(u)$, we shall need the estimates similar to estimates $(22),(23)$ but in the space $\dot{\mathbf{V}}_{2}^{1,0}\left(Q_{T}\right)$. At that, the constants in the obtained inequalities can be obtained explicitly. It gives a chance 
to find explicitly the Lipshitz constant for the gradient $J_{2}^{\prime}(u)$ being important for studying gradient methods of solving extremal problems.

The unique solvability of the problem

$$
\begin{gathered}
L \mathbf{v}=\mathbf{v}_{t}-\nu \Delta \mathbf{v}+g_{i} \mathbf{v}_{x_{i}}=\mathbf{f}-\operatorname{grad} u, \\
\left.\mathbf{v}\right|_{S_{T}}=0,\left.\quad \mathbf{v}\right|_{t=0}=\mathbf{a} .
\end{gathered}
$$

in the space $\stackrel{\circ}{\mathbf{V}}_{2}^{1,0}\left(Q_{t}\right)$ for each $u \in \mathbf{L}_{2}\left(Q_{T}\right), \mathbf{f} \in \mathbf{L}_{2,1}\left(Q_{T}\right)$, a $\in L_{2}(\Omega), g \in \mathbf{L}_{4, \infty}\left(Q_{T}\right)$ follows from [3, Ch. III, Thm. 4.1].

Multiplying equation (29) by $\mathbf{v} e^{-2 \lambda t}$ and differentiating by parts in domain $Q_{t}$, we obtain the identity

$$
\begin{aligned}
\frac{1}{2}\|\widetilde{\mathbf{v}}(t)\|^{2} & +\nu\left\|\left|\widetilde{\mathbf{v}}_{x}\right|\right\|_{0, t}^{2}+\lambda\|\mid \widetilde{\mathbf{v}}\|_{0, t}^{2}+\int_{0}^{t}\left(g_{i} \widetilde{\mathbf{v}}_{x_{i}}, \widetilde{\mathbf{v}}\right) d \tau \\
& =\frac{1}{2}\|\mathbf{a}\|^{2}+\int_{0}^{t}[(\widetilde{f}, \widetilde{\mathbf{v}})+(\widetilde{u}, \operatorname{div} \widetilde{\mathbf{v}})] d \tau
\end{aligned}
$$

where $\widetilde{\mathbf{v}}=\mathbf{v} e^{-\lambda t}, \widetilde{\mathbf{f}}=\mathbf{f} e^{-\lambda t}, \widetilde{u}=u e^{-\lambda t}$.

We consider two cases: the case of bounded functions $g_{i}$ and the case $g \in \mathbf{L}_{4, \infty}\left(Q_{T}\right)$.

Suppose the condition

$$
\max _{i}\left|g_{i}(x, t)\right| \leqslant G \quad \forall(x, t) \in Q_{T},
$$

then

$$
I_{1}=\left|\int_{0}^{t}\left(g_{i} \widetilde{\mathbf{v}}_{x_{i}}, \widetilde{\mathbf{v}}\right) d \tau\right| \leqslant G\left\|\left|\widetilde{\mathbf{v}}_{x_{i}}\right|\left|\left\|_{0, t}\right\|\right| \widetilde{\mathbf{v}}\left|\left\|_{0, t} \leqslant \frac{\nu}{4}\right\|\right| \widetilde{\mathbf{v}}_{x}\left|\left\|_{0, t}^{2}+G^{2} \nu^{-1}\right\|\right| \widetilde{\mathbf{v}} \mid\right\|_{0, t}^{2} .
$$

It is easy to see that the estimates

$$
\begin{gathered}
I_{2}=\left|\int_{0}^{t}(\widetilde{u}, \operatorname{div} \widetilde{\mathbf{v}}) d \tau\right| \leqslant \int_{0}^{t}\|\widetilde{u}\|\left\|\widetilde{\mathbf{v}}_{x}\right\| d \tau \leqslant \frac{\nu}{4}\left\|\widetilde{\mathbf{v}}_{x}\left|\left\|_{0, t}^{2}+\nu^{-1}\right\|\right| \widetilde{u}\right\|_{0, t}^{2}, \\
I_{3}=\left|\int_{0}^{t}(\widetilde{\mathbf{f}}, \widetilde{\mathbf{v}}) d \tau\right| \leqslant \frac{1}{2}\|\widetilde{\mathbf{f}}\|_{0, t}^{2}+\frac{1}{2}\|\widetilde{\mathbf{v}}\|_{0, t}^{2}
\end{gathered}
$$

are valid. Relations (31), (33)-(35) imply the inequality

$$
\begin{aligned}
\frac{1}{2}\|\widetilde{\mathbf{v}}(t)\|^{2} & +\frac{\nu}{2}\left\|\widetilde{\mathbf{v}}_{x}\left|\left\|_{0, t}^{2}+\left[\lambda-\left(G^{2} \nu^{-1}+\nu^{-1}+\frac{1}{2}\right)\right]\right\|\right| \widetilde{\mathbf{v}}\right\|_{0, t}^{2} \\
& \leqslant \frac{1}{2}\|\mathbf{a}\|^{2}+\frac{1}{2}\left\|\widetilde{\mathbf{f}}\left|\left\|_{0, t}^{2}+\nu^{-1}\right\|\right| u \mid\right\|_{0, t}^{2} .
\end{aligned}
$$

Letting $\lambda=G^{2} \nu^{-1}+\nu^{-1}+\frac{1}{2}$, we obtain

$$
\|\widetilde{\mathbf{v}}(t)\|^{2}+\nu\left\|\left|\widetilde{\mathbf{v}}_{x}\right|\right\|_{0, t}^{2} \leqslant\|\mathbf{a}\|^{2}+\left\|\left|\widetilde{\mathbf{f}}\left\|_{0, t}^{2}+2 \nu^{-1}\right\|\right| u \mid\right\|_{0, t} .
$$

It yields the estimate

$$
\|\mathbf{v}\|_{Q_{T}} \leqslant\left(1+\nu^{-\frac{1}{2}}\right) e^{\lambda T}\left(\|\mathbf{a}\|_{L_{2}(\Omega)}^{2}+\|\mathbf{f}\|_{\mathbf{L}_{2}\left(Q_{T}\right)}^{2}+2 \nu^{-1}\|u\|_{\mathbf{L}_{2}\left(Q_{T}\right)}^{2}\right)^{\frac{1}{2}}
$$

where $\lambda=G^{2} \nu^{-1}+\nu^{-1}+\frac{1}{2}$.

If the conditions $\|g\|_{\mathbf{L}_{4, \infty}} \leqslant G$ hold true, to estimate the integral we apply the inequalities

$$
\|v\|_{4} \leqslant 2^{\frac{1}{4}}\left\|v_{x}\right\|^{\frac{1}{2}}\|v\|^{\frac{1}{2}}, \quad n=2, \quad \text { and } \quad\|v\|_{4} \leqslant 2^{\frac{1}{2}}\left\|v_{x}\right\|^{\frac{3}{4}}\|v\|^{\frac{1}{4}}, \quad n=3,
$$


being valid for each $v \in \mathbf{W}_{2}^{1}(\Omega)$, as well as Young's inequality

$$
\left(a b \leqslant \frac{1}{m} \varepsilon_{1}^{m} a^{m}+\frac{m-1}{m} \varepsilon_{1}^{-\frac{m-1}{m}} b^{\frac{m}{m-1}}\right) .
$$

As $n=2$, letting $m=\frac{4}{3}, \varepsilon_{1}=\left(\frac{1}{3} \nu\right)^{\frac{3}{4}}$, we obtain

$$
I_{1} \leqslant 2^{\frac{1}{4}} G\left\|\left|\widetilde{\mathbf{v}}_{x}\right|\right\|_{0, t}^{\frac{3}{2}}\||\mathbf{v}|\|_{0, t}^{\frac{1}{2}} \leqslant \frac{\nu}{4}\left\|\left|\widetilde{\mathbf{v}}_{x}\right|\right\|_{0, t}^{2}+\frac{1}{2}\left(\frac{\nu}{3}\right)^{-\frac{3}{16}} G^{4}\||\widetilde{\mathbf{v}}|\|_{0, t}^{2},
$$

and for $n=3$, letting $m=7, \varepsilon_{1}=\left(\frac{2}{7} \nu\right)^{\frac{7}{8}}$, we get

$$
I_{1} \leqslant 2^{\frac{1}{2}} G\left\|\left|\widetilde{\mathbf{v}}_{x}\right|\right\|_{0, t}^{\frac{7}{4}}\|\| \mathbf{v}\left|\left\|_{0, t}^{\frac{1}{4}} \leqslant \frac{\nu}{4}\right\|\right| \widetilde{\mathbf{v}}_{x}\left|\left\|_{0, t}^{2}+2\left(\frac{2}{7} \nu\right)^{-\frac{7}{64}} G^{8}\right\|\right| \widetilde{\mathbf{v}} \mid \|_{0, t}^{2} .
$$

Due to these estimates, we obtain inequality (36), where

$$
\begin{array}{ll}
\lambda=\lambda_{2}=\frac{1}{2}\left(\frac{\nu}{3}\right)^{-\frac{3}{16}} G^{4}+\nu^{-1}+\frac{1}{2} & \text { as } n=2, \\
\lambda=\lambda_{3}=2\left(\frac{2}{7} \nu\right)^{-\frac{7}{64}} G^{8}+\nu^{-1}+\frac{1}{2} & \text { as } n=3 .
\end{array}
$$

To estimate the adjoint state $\mathbf{w}$, we multiply equation (6) by $\mathbf{w} e^{-2 \lambda(T-t)}$ and integrate by parts over domain $Q_{t}^{T}=\Omega \times[t, T]$. Denoting $\widetilde{\mathbf{w}}=\mathbf{w} e^{-\lambda(T-t)}$, we get

$$
\begin{gathered}
\frac{1}{2}\||\widetilde{\mathbf{w}}|\|^{2}+\nu\left\|\left|\widetilde{\mathbf{w}}_{x}\right|\right\|^{2}+\lambda\||\widetilde{\mathbf{w}}|\|_{0, t}^{2}+\int_{t}^{T}\left(g_{i} \widetilde{\mathbf{w}}, \widetilde{\mathbf{w}}_{x_{i}}\right) d \tau \\
=-\int_{t}^{T}\left(\operatorname{div} v\left(\nabla u e^{-\lambda(T-t)}, \operatorname{div} \widetilde{\mathbf{w}}\right)\right) d \tau .
\end{gathered}
$$

We have obtained relation (31), if we let there $\widetilde{\mathbf{v}}=\widetilde{\mathbf{w}}, \mathbf{a}=0, \widetilde{\mathbf{f}}=0, \widetilde{u}=\operatorname{div} v(\nabla u)$. Thus, we arrive at the estimate

$$
\|\mathbf{w}\|_{Q_{T}} \leqslant\left(1+\nu^{-\frac{1}{2}}\right) e^{\lambda T}\|\operatorname{div} v(\nabla u)\|_{\mathbf{L}_{2}\left(Q_{T}\right)},
$$

where $\lambda=G^{2} \nu^{-1}+\nu^{-1}+\frac{1}{2}$ if $g$ is a bounded function and $\lambda$ is determined by formulae (37) once $g \in \mathbf{L}_{4, \infty}\left(Q_{T}\right)$.

To prove formula $\left(5_{2}\right)$, we can not employ here direct integration by parts since the belonging of functions $v$ and $w$ to space $\mathbf{W}_{2}^{2,1}\left(Q_{T}\right)$ is not guaranteed. We employ the passage to a limit. We choose sequences $u_{n}, h_{n}$ contained in $U_{1}$ such that $u_{n} \longrightarrow u, h_{n} \longrightarrow h$ in $L_{2}\left(Q_{T}\right)$. On sequences $u_{n}, h_{n}$, identities (24) and the first identity in (25) hold true. It implies that

$$
\begin{aligned}
J_{2}\left(u_{n}+h_{n}\right)-J_{2}\left(u_{n}\right) & =-\left(\left(L^{*}\right)^{-1} \nabla \operatorname{div} \mathbf{v}\left(\nabla u_{n}\right), \nabla h_{n}\right)_{\mathbf{L}_{2}\left(Q_{T}\right)}+\frac{1}{2}\left\|\operatorname{div} \mathbf{\mathbf { v }}\left(\nabla h_{n}\right)\right\|_{L_{2}\left(Q_{T}\right)}^{2} \\
& =\left(\operatorname{div} \mathbf{w}\left(u_{n}\right), h_{n}\right)_{L_{2}\left(Q_{T}\right)}+\frac{1}{2}\left\|\operatorname{div} \stackrel{\circ}{\mathbf{v}}\left(\nabla h_{n}\right)\right\|_{L_{2}\left(Q_{T}\right)}^{2} .
\end{aligned}
$$

We denote $\delta h_{n}=h-h_{n}, \delta u_{n}=u-u_{n}, \delta \mathbf{v}_{n}=\mathbf{v}-\mathbf{v}_{n}$, then $\delta \mathbf{v}_{n}$ solves problem (1), (2), where $\mathbf{a}=0, \mathbf{f}=0, u=\delta u_{n}$. Employing estimates (36), (37), we obtain that

$$
\begin{aligned}
& \left\|\operatorname{div} \delta \mathbf{v}_{n}\right\|_{L_{2}\left(Q_{T}\right)} \leqslant c\left\|\delta u_{n}\right\|_{L_{2}\left(Q_{T}\right)}, \\
& \left\|\operatorname{div} \mathbf{w}\left(\delta u_{n}\right)\right\|_{L_{2}\left(Q_{T}\right)} \leqslant c\left\|\delta u_{n}\right\|_{L_{2}\left(Q_{T}\right)} .
\end{aligned}
$$

Passing to the limit in relations (39), we obtain the identity

$$
J_{2}(u+h)-J_{2}(u)=(\operatorname{div} \mathbf{w}(u), h)_{L_{2}(Q)}+\frac{1}{2}\|\operatorname{div} \stackrel{\circ}{\mathbf{v}}(\nabla h)\|_{L_{2}\left(Q_{T}\right)}^{2} .
$$


It follows from estimate $(36)$ that $\|\operatorname{div} \stackrel{\circ}{\mathbf{v}}(\nabla h)\|_{L_{2}\left(Q_{T}\right)}^{2}=O\left(\|h\|_{L_{2}\left(Q_{T}\right)}^{2}\right)$. Hence, formula $\left(5_{2}\right)$ is proven.

Let us show that $J_{2}^{\prime}(u)$ satisfies Lipshitz condition. In order to do it, we employ inequalities (38), (36) to obtain

$$
\begin{aligned}
\left\|J_{2}^{\prime}\left(u^{1}\right)-J_{2}^{\prime}\left(u^{2}\right)\right\|_{L_{2}\left(Q_{T}\right)} & =\left\|\operatorname{div} \mathbf{w}\left(u^{1}\right)-\operatorname{div} \mathbf{w}\left(u^{2}\right)\right\|_{L_{2}\left(Q_{T}\right)}=\left\|\operatorname{div} \mathbf{w}\left(u^{1}-u^{2}\right)\right\|_{L_{2}\left(Q_{T}\right)} \\
& \leqslant \sqrt{n}\left\|\mathbf{w}\left(u^{1}-u^{2}\right)\right\|_{Q_{T}} \leqslant C_{9}\left\|\operatorname{div} \mathbf{v}\left(\nabla\left(u^{1}-u^{2}\right)\right)\right\|_{L_{2}\left(Q_{T}\right)} \\
& \leqslant C_{10}\left\|\mathbf{v}\left(\nabla\left(u^{1}-u^{2}\right)\right)\right\|_{Q_{T}} \leqslant C_{11}\left\|u^{1}-u^{2}\right\|_{L_{2}\left(Q_{T}\right)} .
\end{aligned}
$$

Thus, we have proven the following theorem.

Theorem 3. Let $\boldsymbol{f} \in \boldsymbol{L}_{2}\left(Q_{T}\right), \boldsymbol{g} \in \boldsymbol{L}_{4, \infty}\left(Q_{T}\right)$, div $\boldsymbol{g} \in \boldsymbol{L}_{4, \infty}\left(Q_{T}\right), \boldsymbol{a} \in \stackrel{\circ}{J}\left(Q_{T}\right), S \in C^{2}$, then functional $J_{2}(u)$ is differentiable $L_{2}\left(Q_{T}\right)$ and its gradient satisfies Lipshitz condition.

2.3. Convergence of modified fastest descent method. We shall seek the solution to problems I, II by the gradient projection method (4), where parameter $\alpha_{k+1}$ is chosen by the modified fastest descent method:

$$
\alpha_{k+1}=\min \left[\alpha_{k+1}^{\prime}, \gamma\right] .
$$

Here $\gamma$ is a sufficiently large quantity (the parameter of the method), and $\alpha_{k+1}^{\prime}$ is defined as in the fastest descent method

$$
f_{k}\left(\alpha_{k+1}^{\prime}\right)=\min _{\alpha>0} f_{k}(\alpha), f_{k}(\alpha)=J\left(P_{U}\left(u_{k}-\alpha J^{\prime}\left(u_{k}\right)\right)\right) .
$$

Since the suggest method can be also employed in other optimization problem, where set $U$ is the whole space or a subspace, we formulate the statement as a theorem in an abstract Hilbert space $H$.

We introduce the notations $J_{*}=\inf _{U} J(u), U_{*}=\left\{u \in U: J(u)=J_{*}\right\}, C^{1,1}(u)$ is the set of differentiable functionals whose gradient satisfy Lipshitz condition.

Theorem 4. Let $U$ be a convex closed set in a Hilbert space $H, J(u) \in C^{1,1}(u)$ be a convex functional. Suppose that the set $U_{*}$ is non-empty and bounded, the sequence $\left\{u_{k}\right\}_{k=0}^{\infty}$ is defined by formula (4) and the conditions

$$
\begin{gathered}
\sum_{k=0}^{\infty}\left\|J^{\prime}\left(u_{k}\right)\right\|^{2} \leqslant b_{1}, \\
0<\alpha_{k}<b_{2}
\end{gathered}
$$

hold true. Then sequence $\left\{u_{k}\right\}_{k=0}^{\infty}$ minimize function $J(u)$ on $U$ converges to set $U_{*}$ weakly in $H$.

Proof. Denote $\rho\left(u, U_{*}\right)=\min _{v \in U_{*}}\|u-v\|$, then by the definition of the projection operator

$$
\begin{aligned}
\rho^{2}\left(u_{k+1}, U_{*}\right) & =\left\|u_{k+1}-P_{U_{*}}\left(u_{k+1}\right)\right\|^{2} \leqslant\left\|u_{k+1}-P_{U_{*}}\left(u_{k}\right)\right\|^{2} \\
& =\left\|P_{U}\left(u_{k}-\alpha_{k+1} J^{\prime}\left(u_{k}\right)\right)-P_{U}\left(P_{U_{*}}\left(u_{k}\right)\right)\right\|^{2} \leqslant \\
& \leqslant\left\|u_{k}-\alpha_{k+1} J^{\prime}\left(u_{k}\right)-P_{U_{*}}\left(u_{k}\right)\right\|^{2} \\
& =\rho^{2}\left(u_{k}, U_{*}\right)+\alpha_{k+1}^{2}\left\|J^{\prime}\left(u_{k}\right)\right\|^{2}-2 \alpha_{k+1}\left(J^{\prime}\left(u_{k}\right), u_{k}-P_{U_{*}}\left(u_{k}\right)\right) .
\end{aligned}
$$

Employing the criterion for the convexity of a differentiable functional on a convex set $U$

$$
J(u)-J(v) \geqslant\left(J^{\prime}(v), u-v\right) \quad \forall u, v \in U,
$$

and letting $v=u_{k}, u=P_{U_{*}}\left(u_{k}\right)$, we obtain

$$
0 \leqslant J\left(u_{k}\right)-J\left(P_{U_{*}}\left(u_{k}\right)\right)=J\left(u_{k}\right)-J_{*} \leqslant\left(J^{\prime}\left(u_{k}\right), u_{k}-P_{U_{*}}\left(u_{k}\right)\right) .
$$


Thus, we get

$$
\left(J^{\prime}\left(u_{k}\right), u_{k}-P_{U_{*}}\left(u_{k}\right)\right) \geqslant J\left(u_{k}\right)-J_{*} \geqslant 0 .
$$

Taking into consideration the inequalities (43), (44), we have

$$
\rho^{2}\left(u_{k+1}, U_{*}\right)-\rho^{2}\left(u_{k}, U_{*}\right) \leqslant \alpha_{k+1}^{2}\left\|J^{\prime}\left(u_{k}\right)\right\|^{2} .
$$

Summing up the last inequality from 0 to $m>0$ and bearing in mind condition (41), we arrive at

$$
\rho^{2}\left(u_{m}, U_{*}\right) \leqslant \sum_{k=0}^{m} \alpha_{k+1}^{2}\left\|J^{\prime}\left(u_{k}\right)\right\|^{2}+\rho^{2}\left(u_{0}, U_{*}\right) \leqslant b_{2}^{2} b_{1}+\rho^{2}\left(u_{0}, U_{*}\right)=b_{3} .
$$

Thus, sequence $\left\{u_{k}\right\}_{k=0}^{\infty}$ is bounded in $H$, and condition (41) follows that $\lim _{k \rightarrow \infty}\left\|J^{\prime}\left(u_{k}\right)\right\|=0$. Now inequality (44) implies that sequence $\left\{u_{k}\right\}_{k=0}^{\infty}$ minimizes functional $J(u)$. Thus, sequence $\left\{u_{k}\right\}_{k=0}^{\infty}$ is bounded and minimizing $J(u)$ on $U$.

We denote by $W$ the set of convex combination of sequence $\left\{u_{k}\right\}_{k=0}^{\infty}$, i.e., the set of points $u$ represented as

$$
u=\sum_{k=0}^{\infty} \alpha_{k} u_{k}, \quad \alpha_{k} \geqslant 0 \quad k=0,1, \ldots, \quad \sum_{k=0}^{\infty} \alpha_{k}=1 .
$$

Employing [8, Ch. 4, Sec. 8, Thm. 5], it is easy to show that $W \subset U$ and since $U$ is a closed set, the closure $\bar{W}$ of set $W$ belongs to $U$ as well.

Sequence $\left\{u_{k}\right\}_{k=0}^{\infty}$ minimizes function $J(u)$ on $U$ and thus minimizes $J(u)$ on $\bar{W}$. It follows that $J_{*}(\bar{W})=\inf _{u \in \bar{W}} J(u)=J_{*}=\inf _{u \in U} J(u), \bar{W}_{*}=\left\{u \in \bar{W}: J(u)=J_{*}\right\} \in U_{*}$. The boundedness of sequence $\left\{u_{k}\right\}_{k=0}^{\infty}$ follows boundedness of set $\bar{W}$. In accordance with [8, Ch. 1, Sec. 3, Thm. 6], a convex lower semibounded functional $J(u)$ on a bounded convex closed set $U$ in a reflexive Banach space has a non-empty set of minimum points $U_{*}$ and each minimizing sequence $\left\{u_{k}\right\}_{k=0}^{\infty}$ converges weakly to $U_{*}$. The weak convergence of sequence $\left\{u_{k}\right\}_{k=0}^{\infty}$ to $\bar{W}_{*}$ implies its weak convergence to $U_{*}$. The proof is complete.

Remark 1. If set $\bar{W}$ is compact, the strong convergence holds true. Here we can apply Theorem 1 in [8, Ch. 1, Sec. 3]).

Remark 2. If $U$ is a subspace of a Hilbert space $H, P_{U}$ is the orthogonal projector on this space, then $u_{k+1}=u_{k}-P_{U} J^{\prime}\left(u_{k}\right)$. In this case relation (43) can be written as

$$
\rho^{2}\left(u_{k+1}, U_{*}\right)=\rho^{2}\left(u_{k}, U_{*}\right)+\alpha_{k+1}^{2}\left\|P_{U} J^{\prime}\left(u_{k}\right)\right\|^{2}-2 \alpha_{k+1}\left(P_{U} J^{\prime}\left(u_{k}\right), u_{k}-P_{U_{*}}\left(u_{k}\right)\right) .
$$

In view of the identity $\left(P_{U} J^{\prime}\left(u_{k}\right), u_{k}-P_{U_{*}}\left(u_{k}\right)\right)=\left(J^{\prime}\left(u_{k}\right), u_{k}-P_{U_{*}}\left(u_{k}\right)\right)$ it is easy to see that the statement of the theorem holds if one replaces condition (41) by the condition

$$
\sum_{k=0}^{\infty}\left\|P_{U} J^{\prime}\left(u_{k}\right)\right\|^{2}<b_{1}
$$

Since sets $U_{l}, l=1,2$, are the subspaces of corresponding spaces and thus the projectors $P_{l}$ on these sets are linear, let us find the explicit formulae for the parameters $\alpha_{k+1}^{\prime}, \alpha_{k+1}$.

Indeed,

$$
\begin{aligned}
f_{l, k}(\alpha)= & J_{l}\left(\mathbf{v}\left(P_{l}\left(u_{k}-\alpha J_{l}^{\prime}\left(u_{k}\right)\right)\right)\right)=\frac{1}{2}\left\|\mid \operatorname{div} \mathbf{v}\left(u_{k}-\alpha P_{l} J_{l}^{\prime}\left(u_{k}\right)\right)\right\|_{0, T}^{2} \\
= & \frac{1}{2}\left\|\left|\operatorname{div} \mathbf{v}\left(u_{k}\right)\right|\right\|_{0, T}^{2}-2 \alpha\left(\operatorname{div} \mathbf{v}\left(u_{k}\right), \operatorname{div} \stackrel{\circ}{\mathbf{v}}\left(P_{l} J_{l}^{\prime}\left(u_{k}\right)\right)\right)_{\mathbf{L}_{2}\left(Q_{T}\right)} \\
& +\alpha^{2}\left\|\left|\operatorname{div} \stackrel{\circ}{\mathbf{v}}\left(P_{l} J_{l}^{\prime}\left(u_{k}\right)\right)\right|\right\|_{0, T}^{2} .
\end{aligned}
$$

It follows that

$$
\alpha_{k}^{\prime}=\left(\operatorname{div} \mathbf{v}\left(u_{k}\right), \operatorname{div} \stackrel{\circ}{\mathbf{v}}\left(P_{l} J_{l}^{\prime}\left(u_{k}\right)\right)\right)_{\mathbf{L}_{2}\left(Q_{T}\right)}\left\|\left|\operatorname{div} \stackrel{\circ}{\mathbf{v}}\left(P_{l} J_{l}^{\prime}\left(u_{k}\right)\right)\right|\right\|_{0, T}^{-2}
$$


Here the expressions $\mathbf{v}\left(u_{k}\right), \stackrel{\circ}{\mathbf{v}}\left(P_{l} J_{l}^{\prime}\left(u_{k}\right)\right)$ mean $\mathbf{v}\left(\nabla u_{k}\right), \stackrel{\circ}{\mathbf{v}}\left(\nabla P_{l} J_{l}^{\prime}\left(u_{k}\right)\right)$, where $\mathbf{v}(u)$ is the solution to equation (1) for $f=0$ and $\mathbf{a}=0$.

It is clear that sequences $\left\{J_{l}\left(u_{k}\right)\right\}_{k=0}^{\infty}$ are monotonically decreasing and are bounded from below.

It was shown in the previous subsection that $J_{l}(u) \in C^{1,1}\left(U_{l}\right)$. We then apply a known estimate being valid for the functions in $C^{1,1}(u)$, see [8, Eq. (2.3.7)]

$$
\left|J(u)-J(v)-\left(J^{\prime}(v), u-v\right)\right| \leqslant \frac{L}{2}\|u-v\|^{2} \quad \forall u, v \in U,
$$

where $L$ is the Lipshitz constant. Letting here $v=u_{k}, u=u_{k+1}^{\alpha}=u_{k}-\alpha P_{l} J_{l}^{\prime}\left(u_{k}\right)$, we obtain

$$
\begin{aligned}
J_{l}\left(u_{k}\right)-J_{l}\left(u_{k+1}^{\alpha}\right) & =J_{l}\left(u_{k}\right)-J_{l}\left(u_{k}-\alpha P_{l} J_{l}^{\prime}\left(u_{k}\right)\right) \\
& \geqslant \alpha\left(J_{l}^{\prime}\left(u_{k}\right), P_{l} J_{l}^{\prime}\left(u_{k}\right)\right)_{H_{l}}-\frac{L_{l}}{2} \alpha^{2}\left\|P_{l} J_{l}^{\prime}\left(u_{k}\right)\right\|^{2},
\end{aligned}
$$

where $L_{l}$ is the Lipshitz constant for the gradient $J_{l}^{\prime}(u)$ of functional $J_{l}(u)$. Taking into account that $P_{l}$ is the orthogonal projector on a subspace, we obtain that $\left(J_{l}^{\prime}\left(u_{k}\right), P_{l} J_{l}^{\prime}\left(u_{k}\right)\right)_{H_{l}}=$ $\left\|P_{l} J^{\prime}\left(u_{k}\right)\right\|_{H_{l}}$. Then it follows from inequality (48) that

$$
J_{l}\left(u_{k}\right)-J_{l}\left(u_{k+1}^{\alpha}\right) \geqslant \alpha\left(1-\alpha \frac{L_{l}}{2}\right)\left\|P_{l} J_{l}^{\prime}\left(u_{k}\right)\right\|_{H_{l}}^{2} .
$$

Letting $\alpha=1 / L_{l}$, we obtain

$$
J_{l}\left(u_{k}\right)-J_{l}\left(u_{k+1}^{\alpha}\right) \geqslant 1 / 2 L_{l}\left\|P_{l} J_{l}^{\prime}\left(u_{k}\right)\right\|_{H_{l}}^{2} .
$$

Suppose that $\alpha_{k+1}^{\prime} \leqslant \gamma$, then $\alpha_{k+1}=\alpha_{k+1}^{\prime}$. Thus, for $\alpha=1 / L_{l}$ the inequalities

$$
J_{l}\left(u_{k}\right)-J_{l}\left(u_{k+1}\right) \geqslant J_{l}\left(u_{k}\right)-J\left(u_{k+1}^{\alpha}\right) \geqslant 1 / 2 L_{l}\left\|P_{l} J_{l}^{\prime}\left(u_{k}\right)\right\|_{H_{l}}^{2}
$$

hold true.

Suppose now that $\alpha_{k+1}^{\prime}>\gamma$, then $\alpha_{k+1}=\gamma$. We consider two cases: $\gamma \geqslant 1 / L_{l}$ and $\gamma<1 / L_{l}$. Since function $f_{l, k}(\alpha)$ decreases on the interval $\left(0, \alpha_{k}^{\prime}\right)$, in the first case we obtain inequalities (50). In the second case $\left(\gamma<1 / L_{l}\right.$

$$
\gamma\left(1-\gamma L_{l} / 2\right) \geqslant \frac{1}{2} \gamma
$$

Thus, due to inequalities (49), (50), in all cases we obtain the estimate

$$
J_{l}\left(u_{k}\right)-J_{l}\left(u_{k+1}\right) \geqslant c_{l}\left\|P_{l} J_{l}^{\prime}\left(u_{k}\right)\right\|_{H_{l}}^{2},
$$

where $c_{l}=\min \left[\frac{1}{2} \gamma, \frac{1}{2} / L_{l}\right]$. The last estimate yields that the sequence $\left\{J_{l}\left(u_{k}^{l}\right)\right\}_{k=0}^{\infty}$ decreases monotonically, the series $\sum_{k=0}^{\infty}\left\|P_{l} J_{l}^{\prime}\left(u_{k}\right)\right\|_{H_{l}}^{2}$ converges and the estimate

$$
\sum_{j=k}^{\infty}\left\|P_{l} J_{l}^{\prime}\left(u_{k}^{l}\right)\right\|_{H_{l}}^{2} \leqslant c_{l}^{-1}\left(J_{l}\left(u_{k}\right)-J_{l, *}\right)
$$

holds true, where $J_{l, *}=\inf _{u \in U_{l}} J_{l}(u)$. Thus, the gradients $J_{l}^{\prime}(u)$ of functionals $J_{l}(u), l=1,2$, satisfy inequality $\left(41^{\prime}\right)$.

It is easy to make sure that functional $J_{l}(u), l=1,2$, are convex. Indeed, for each $\alpha \in[0,1]$

$$
\begin{aligned}
& J_{l}(\alpha u+(1-\alpha) w)=\|\alpha \operatorname{div} \mathbf{v}(u)+(1-\alpha) \operatorname{div} \mathbf{v}(w)\|_{0, T}^{2} \\
& \quad=\alpha^{2}\|\operatorname{div} \mathbf{v}(u)\|_{0, T}^{2}+(1-\alpha)^{2}\|\operatorname{div} \mathbf{v}(w)\|_{0, T}^{2}+2 \alpha(1-\alpha)(\operatorname{div} \mathbf{v}(u), \operatorname{div} \mathbf{v}(w))_{\mathbf{L}_{2}\left(Q_{T}\right)} \\
& \quad=\alpha\|\operatorname{div} \mathbf{v}(u)\|_{0, T}^{2}+(1-\alpha)\|\operatorname{div} \mathbf{v}(w)\|_{0, T}^{2}-\alpha(1-\alpha)\|\operatorname{div} \mathbf{v}(u)-\operatorname{div} \mathbf{v}(w)\|_{0, T}^{2} \\
& \quad \leqslant \alpha J_{l}(u)+(1-\alpha) J_{l}(w) .
\end{aligned}
$$


Taking into consideration Remark 2 for Theorem 4, Theorems 2 and 3 on differentiability of functionals $J_{l}(u), l=1,2$, and the well-known theorems on the unique solvability of problem (1)-(3) [1, Ch. 4. Sec. 1, Thms. 1', 2], one can easily prove the following theorem.

Theorem 5. Let $\boldsymbol{f} \in \boldsymbol{L}_{2}\left(Q_{T}\right), \boldsymbol{g} \in \boldsymbol{L}_{4, \infty}\left(Q_{T}\right)$, div $\boldsymbol{g} \in L_{4, \infty}\left(Q_{T}\right)$, a $(x)$ satisfies condition (1.4), $S \in C^{2}$. Then the sequence $\left\{u_{k}^{l}\right\}_{k=0}^{\infty}$ defined by identities (4), $\left(5_{l}\right), l=1,2$, where parameter $\alpha_{k+1}$ is defined by (40), (47), minimizes functional $J_{l}(u)$ on $U_{l}$ and converges to $U_{l, *}$ weakly in $H_{l}$ from each initial approximation.

Remark. As $l=1$, Theorem 5 is implied immediately by Theorem 4 since the hypothesis of this theorem holds true. As $l=2$, the unique solvability of problem (1)-(3) does not ensure one of the conditions of Theorem 5 that $U_{*}$ is non-empty and bounded. However under the hypothesis of Theorem 5 there exists the unique solution to problem (1)-(3) in the class $\mathbf{v} \in \mathbf{W}_{2}^{2,1}\left(Q_{T}\right), p \in \mathbf{W}_{2}^{1,0}\left(Q_{T}\right)$. It is clear this solution is also that of the generalized problem, while the solution to the generalized problem is unique. Thus, functional $J_{2}(u)$ in the hypothesis of Theorem 5 satisfies all the assumptions of Theorem 4 and moreover, in this case the condition $\operatorname{div} g \in L_{4, \infty}\left(Q_{T}\right)$ can be neglected.

2.4. Regularization of iteration process by Tikhonov method. In the previous subsection we have proven the weak convergence of the modified fastest descent method for functionals $J_{l}(u), l=1,2$.

In order to construct strongly converging sequence one can employ the Tikhonov regularization method [8]. Its matter is the consecutive solving of the minimization problems for functionals $T_{j}(u)=J(u)+\beta_{j} \Omega(u)$ on $U$ as first kind problems, i.e., the problem on minimization w.r.t. the functional. Here $\Omega(u)$ is the stabilizer or a non-negative strongly convex function. For a fixed $j$ we find a point $u_{j}$ satisfying the conditions

$$
T_{j}^{*}=\underset{U}{\inf } T_{j}(u) \leqslant T_{j}\left(u_{j}\right)<T_{j}^{*}+\varepsilon_{j} .
$$

Tikonov's theorem (see, for instance, [8, Ch. 2, Sec. 5, Thm. 1]) implies that if $J(u) \in$ $C^{1,1}(u), U_{*}$ is non-empty, $J_{*}>-\infty$,

$$
\lim _{j \rightarrow \infty} \beta_{j}=\lim _{j \rightarrow \infty} \varepsilon_{j}=0, \quad \sup _{j>1} \varepsilon_{j} \beta_{j}^{-1}<\infty,
$$

then the sequence $\left\{u_{j}\right\}_{j=1}^{\infty}$ defined by conditions (53) minimizes functional $J(u)$ on $U$ and $\lim _{j \rightarrow \infty} \rho\left(u_{j}, U_{*}\right)=0$.

Returning back to the original problem (1)-(3), we introduce the notations

$$
T_{l, j}(u)=J_{l}(u)+\beta_{j}\|u\|_{H_{l}}^{2}, u \in U_{l}, \beta_{j}>0, \lim _{j \rightarrow \infty} \beta_{j}=0 .
$$

Since for $\beta_{j}>0$ functional $T_{l, j}(u), l=1,2$, is strongly convex, it has the unique minimum point $u_{l, j}^{*}$. In what follows, if the arguments are same, we omit the subscript $l$, at that, $\|\cdot\|=\|\cdot\|_{H_{l}}$.

For the approximate solving of the minimization problem for functional $T_{l, j}(u)$ we employ the usual fastest descent method

$$
u_{j, k+1}=P_{U_{l}}\left(u_{j, k}-\alpha_{j, k+1} T_{l, j}^{\prime}\left(u_{j, k}\right)\right), j=1,2, \ldots ; k=0,1, \ldots, n_{j} .
$$

Parameter $\alpha_{j, k+1}$ is calculated explicitly by the formula

$$
\begin{aligned}
\alpha_{j, k+1}= & {\left[\left(\operatorname{div} \mathbf{v}\left(u_{j, k}\right), \operatorname{div} \stackrel{\circ}{\mathbf{v}}\left(P_{l} T_{l, j}^{\prime}\left(u_{j, k}\right)\right)\right)_{\mathbf{L}_{2}\left(Q_{T}\right)}+\beta_{j}\left(u_{j, k}, P_{l} T_{l, j}^{\prime}\left(u_{j, k}\right)\right)_{H_{1}}\right] } \\
& \times\left[\left\|\operatorname{div} \stackrel{\circ}{\mathbf{v}}\left(P_{l} T_{l, j}^{\prime}\left(u_{j, k}\right)\right)\right\|_{\mathbf{L}_{2}\left(Q_{T}\right)}^{2}+\beta_{j}\left\|P_{l} T_{l, j}^{\prime}\left(u_{j, k}\right)\right\|_{H_{1}}^{2}\right]^{-1} .
\end{aligned}
$$

As in formula (47), here for $l=1,2$

$$
\mathbf{v}\left(u_{j, k}\right)=\mathbf{v}\left(\nabla u_{j, k}\right) ; \stackrel{\circ}{\mathbf{v}}\left(P_{l} T_{l, j}^{\prime}\left(u_{j, k}\right)\right)=\stackrel{\circ}{\mathbf{v}}\left(\nabla P_{l} T_{l, j}^{\prime}\left(u_{j, k}\right)\right) .
$$


Let $\beta_{j} \rightarrow 0$ as $j \rightarrow \infty$ and $\beta_{j}>0$. For each fixed $j$, we make $n_{j}$ iteration by scheme (56) and as the initial value for the minimization of functional $T_{l, j+1}$ we take $u_{j+1,0}=u_{j}=u_{j, n_{j}}$. We choose $n_{j}$ by the condition

$$
T_{l, j}^{\prime}\left(u_{j, n_{j}}\right) \leqslant \beta_{j}
$$

Let us show that in this case sequence $\left\{u_{j}\right\}$ satisfies conditions (53), (54) of Tikhonov's theorem. We take into consideration the well-known inequality for strongly convex functionals

$$
J(u)-J\left(u_{*}\right) \leqslant \frac{1}{2 \mu}\left\|J^{\prime}(u)\right\|^{2},
$$

where $\mu$ is the constant from the criterion of strong convexity of a functional

$$
\left(J^{\prime}(u)-J^{\prime}(v), u-v\right) \geqslant \mu\|u-v\| \quad \forall u, v \in U .
$$

In the considered case $\mu \geqslant \beta_{j}$. It follows from inequalities (58), (59) that

$$
T_{l, j}\left(u_{j}\right)-T_{l, j}\left(u_{l, j}^{*}\right) \leqslant \frac{1}{2} \beta_{j}=\varepsilon_{j}
$$

Thus, the hypothesis of Tiknonov's theorem holds true. Taking into consideration that the minimum of functionals $J_{l}(u), l=1,2$ under the hypothesis of Theorem 5 is equal to zero and it is attained at the only point being the solution to problem (1)-(3), we arrive at the following theorem.

Theorem 6. Suppose the hypothesis of Theorem 5. Then sequence $\left\{u_{j}\right\}_{j=1}^{\infty}$ defined by relations (56)-(58) minimizes functional $J_{l}(u)$ on $U_{l}$ and

$$
\lim _{j \rightarrow \infty}\left\|u_{j}-u_{*}\right\|_{H_{l}}=0 .
$$

Remark. Let $\mathbf{v}, p$ be the solution to problem (1)-(3), then $u_{*}=p$. Taking into consideration that the norm $\|\cdot\|_{\lambda}$ is equivalent to

$$
\|\mathbf{v}\|=\underset{t \in[0, T]}{\operatorname{vraimax}}\left\|\mathbf{v}_{x}\right\|+\|\Delta \mathbf{v}\|_{\mathbf{L}_{2}\left(Q_{T}\right)}+\left\|\mathbf{v}_{x}\right\|_{\mathbf{L}_{2}\left(Q_{T}\right)}
$$

and estimates (22) and (36), we obtain the relations

$$
\lim _{j \rightarrow \infty}\left\|\mathbf{v}_{j}^{1}-\mathbf{v}\right\|=0, \quad \lim _{j \rightarrow \infty}\left\|\mathbf{v}_{j}^{2}-\mathbf{v}\right\|_{Q_{T}}=0,
$$

where $\left\{\mathbf{v}_{j}^{1}\right\}$ is the sequence defined in solving Problem I, while $\left\{\mathbf{v}_{j}^{2}\right\}$ is the sequence defined in solving Problem II.

\section{Conclusion}

In the present work we suggest an approach for solving problem (1.1)-(1.3). The matter of the approach is a consecutive solving of linearized problems by the gradient method. We note that in this case the minimizing functional is convex. One can also employ another approach where problem (1.1)-(1.3) is regarded as the inverse problem, where relations (1.1)-(1.3) describes the state of a system under an unknown pressure, while identity (1.3) introduces additional data on the state of a system. This problem is easily formulated as a problem of optimal control:

$$
J(p)=\int_{Q_{T}}|\operatorname{div} \mathbf{v}(p)|^{2} d x d t \rightarrow \inf ; \quad p \in U_{l}, \quad l=1,2,
$$

where $\mathbf{v}(p)$ solves problem (1.1), (1.2) for a given $p \in U_{l}$.

Employing the obtained or apriori restrictions for the speed vector $\mathbf{v}$, we can replace equation (1.1) by equation $\left(1.1^{\prime}\right)$ and justify the unique solvability for problem $\left(1.1^{\prime}\right),(1.2)$ as well as the convergence of the iteration process (1.5), (1.6) for each fixed $p \in U_{l}, l=1,2$. The construction and justification of the gradient method for solving problem (1.1)-(1.3) in such formulation as well as the comparison of various ways of numerical realization of the suggested methods will be given in a future work. One of the options was tested on a model example where the 
solution to problem (1.1)-(1.3) and thus the apriori estimate were known. The calculations were made consecutively by time layers. In this case to obtain a given precision 3-4 steps of iterative linearization was needed and 5-6 steps of gradient descent.

\section{BIBLIOGRAPHY}

1. O.A. Ladyženskaya. Mathematical problems in the dynamics of a viscous incompressible fluid. 2ed. Moscow, Nauka, 1970. [1ed. Gordon and Breach, New York, 1963.]

2. R. Temam. Navier-Stokes Equations. Theory and Numerical Analysis. North-Holland, Amsterdam, 1977.

3. I.I. Golichev. Iterative linearization of the evolution Navier-Stokes equations // Ufimskij Matem. Zhurn. 2012. V. 4, No. 4. P. 69-78. [Ufa Math. J. 2012. V. 4, No. 4. P.68-77.]

4. O.A. Ladyženskaja, V.A. Solonnikov, N.N. Ural'ceva. Linear and quasi-linear equations of parabolic type. Nauka, Moscow, 1967. [Trans. Math. Monographs. V. 23. Amer. Math. Soc., Providence, RI, 1968.]

5. V.I. Agoshkov, E.A. Botvinovskii. Numerical solution of the nonstationary Stokes system by methods of adjoint-equation theory and optimal control theory // Zhurn. vychisl. matem. matem. fiz. 2007. V. 47, No. 7. P. 1192-1207. [Comp. Math. Math. Phys. 2007. V. 47, No. 7. P. 1142-1157.]

6. I.I. Golichev, T.R. Sharipov. Development of methods, algorithms and programs for solving NavierStokes equations as problem of optimal control // Vestnik UGATU. Matem. 2007. V. 9, No. 3(21). P. 51-57. (in Russian.)

7. I.I. Golichev. Gradient methods for solving Navier-Stokes equations // Obozrenie Prikladnoj i Promyshlennoj Matem. 2001. V. 18, No. 3. P. 423-425. (in Russian.)

8. F.P. Vasil'ev. Methods for solving extremal problems. Moscow, Nauka, 1981. (in Russian.)

9. F.P. Vasil'ev. Numerical methods for solving extremal problems. Nauka, Moscow, 1988. (in Russian.)

Golichev Iosif Iosifovich, Institute of Mathematics USC RAS,

Chernyshevskogo str., 112,

450008, Ufa, Russia

E-mail: Golichev ii@mail.ru 